\title{
Editorial
}

\section{Reflexiones conceptuales y metodológicas sobre la Metasíntesis}

\section{Reflexões conceituais e metodológicas sobre Metassíntese \\ Conceptual and methodological reflections on Metasynthesis}

\author{
César Hueso Montoro \\ Profesor del Departamento de Enfermería de la Universidad de Granada. \\ Cómo citar esta editorial en edición digital: Hueso Montoro, C. (2016). Reflexiones conceptuales y metodológicas sobre la \\ Meta-síntesis. Cultura de los Cuidados (Edición digital),20(46). \\ Disponible en: <http://dx.doi.org/10.14198/cuid.2016.46.01> \\ Correspondencia: César Hueso Montoro. Facultad de Ciencias de la Salud, Universidad de Granada. \\ Avenida de la Ilustración 60, 18016, Granada, España \\ Correo electrónico: cesarhueso@ugr.es, twitter: @chuesomontoro
}

Recibido: Autor invitado

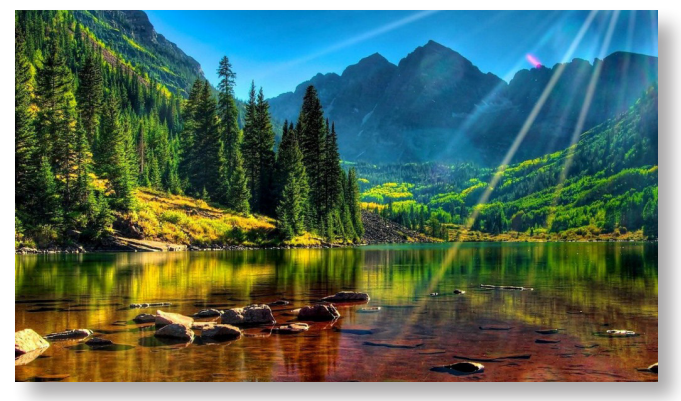

ABSTRACT

The aim of this editorial is to reflect on the concept of "metasynthesis". Although there is some heterogeneity when referring to metasynthesis, there is some consensus in talking about Metasynthesis. The most essential interpretation is the one that describes it as a secondary research procedure, whose purpose is to review and synthesize rigorously and with a critical approach the knowledge coming from original research developed with qualitative methodology.

Keywords: Metasynthesis, qualitative research, secondary research, practice based on evidence.

\section{RESUMO}

O objetivo deste editorial é refletir sobre o conceito de metasíntese. Embora exista alguma heterogeneidade al referir-se a uma metástase, o que existe é consenso em falar da Metasíntesis. A interpretação mais essencial é a que descrevem como um procedimento de investigação secundária, cujo propósito é revisar e sintetizar de forma rigurosa e com enfoque crítico o conhecimento procedente de investigações originais desenvolvidas com metodologia qualitativa.

Palavras chave: Metasíntesis, pesquisa cualitativa, pesquisa secundária, prática baseada na evidênci.

\section{RESUMEN}

El objetivo de esta editorial consiste en reflexionar sobre el concepto de "metasíntesis". Aunque existe alguna heterogeneidad al referirse a metasíntesis, sí que hay cierto consenso en hablar de la Metasíntesis. La interpretación más esencial es la que la describe como un procedimiento de investigación secundaria, 
cuyo propósito es revisar y sintetizar de manera rigurosa y con enfoque crítico el conocimiento procedente de investigaciones originales desarrolladas con metodología cualitativa.

Palabras clave: Metasíntesis, investigación cualitativa, investigación secundaria, práctica basada en la evidencia.

El movimiento de la Práctica Basada en la Evidencia (PBE) ha potenciado estrategias metodológicas de investigación secundaria que se sustentan en síntesis exhaustivas y críticas del conocimiento científico disponible. Uno de sus máximos exponentes son las revisiones sistemáticas de la Cochrane, centradas fundamentalmente en ofrecer las mejores evidencias disponibles basadas en síntesis de hallazgos procedentes de estudios experimentales. La investigación cualitativa no ha permanecido ajena a esta realidad, de modo que también es habitual encontrar en la literatura científica la publicación de estudios de síntesis del conocimiento elaborado a partir de diseños cualitativos. A pesar de la heterogeneidad de conceptos que se utilizan a este respecto, hay cierto consenso en hablar de la Metasíntesis para referirnos a ello, entendida como un procedimiento de investigación secundaria cuyo propósito es revisar y sintetizar de manera rigurosa y con enfoque crítico, el conocimiento procedente de investigaciones originales desarrolladas con metodología cualitativa (Carrillo-González, Gómez-Ramírez \& Vargas-Rosero, 2007).

\section{¿Qué aportan estas metodologías?}

En primer lugar, suponen una oportunidad para el desarrollo de líneas de investigación centradas en la perspectiva tanto de pacientes como de profesionales, como así consta en trabajos ya publicados (Blanca-Gutiérrez,
Linares-Abad, Grande-Gascón, Jiménez-Díaz \& Hidalgo-Pedraza, 2012; Boyce, Browne \& Greenhalgh, 2014; Balmer, Griffiths \& Dunn, 2014; Chen \& Chang 2015; Hernández Zambrano, Hueso Montoro, Montoya Juárez, Gómez Urquiza \& Bonill de las Nieves 2016), pero que incorporan a su vez una visión transversal del problema en diferentes contextos sociales. El hecho de revisar estudios realizados en diferentes contextos, garantiza de algún modo que los resultados finales dimensionen el fenómeno investigado con perspectiva universal, lo cual aminora los problemas que a este respecto generan los estudios cualitativos originales. $\mathrm{Si}$ bien es cierto que estos procedimientos a veces no representan la singularidad de determinados espacios que pueden influir notablemente en la interpretación que se haga de los resultados allí generados.

En segundo lugar, y muy en relación con lo anterior, la Metasíntesis ofrece una oportunidad para dar recorrido a los valores y expectativas de los pacientes en el marco de la $\mathrm{PBE}$, que supone uno de los pilares conceptuales de este movimiento. Así, los profesionales clínicos pueden obtener de estos estudios elementos clave para comprender la experiencia del paciente e incorporarlos en sus decisiones clínicas. El nivel de recomendación conocido como evidencia $\mathrm{Q}$ cada vez está más presente en Guías de Prácticas Clínica y otros documentos de interés clínico (Luengo, 2010).

A colación con lo anterior, el Grupo Cochrane de Métodos de Investigación Cualitativa propone diferentes aspectos que la investigación cualitativa podría aportar en las clásicas revisiones de intervenciones: pueden ayudar a definir o replantear la pregunta, contextualiza la efectividad de las intervenciones revisadas $y$ propone preguntas que no necesariamente tienen que ver con la efectividad, pero sí pueden 
ser pertinentes en la práctica clínica (Higgins \& Green, 2011).

\section{Algunas reflexiones metodológicas}

Desde el año 2012, el grupo de investigación "Aspectos Psicosociales y Transculturales de la Salud y la Enfermedad" de la Universidad de Granada (CTS-436) en colaboración con la Fundación Index, viene desarrollando una metasíntesis sobre la experiencia de personas portadoras de estomas digestivos a través de un proyecto financiado por la Consejería de Salud de la Junta de Andalucía (Capilla Díaz et al, 2014). Se han revisado estudios hasta 2015 y ya se han publicado los primeros resultados (Capilla Díaz et al, 2016). La experiencia de estos años ha permitido un acercamiento a esta metodología tanto en el plano conceptual como metodológico, por lo que se proponen a continuación algunas reflexiones que son producto de las decisiones que se han ido tomando en el transcurso del proyecto. No se pretende hacer un manual metodológico al respecto, para lo que se recomiendan otras lecturas más amplias (Mahtani, Axpe, Serrano, González \& Fernández, 2007), sino simplemente se quiere hacer énfasis en algunos aspectos que son clave en el desarrollo de estos estudios y que pueden ayudar a otros investigadores interesados en estos procedimientos.

¿Qué fuentes documentales utilizar?

Las Bases de Datos Bibliográficas suponen la fuente de elección por excelencia. Ofrecen los registros estructurados por campos y esto facilita enormemente los pasos de selección de los trabajos a incluir finalmente en la revisión. Además, la mayoría permiten filtrar la búsqueda por tipo de investigación, de modo que bases de datos como PUBMED o CINAHL permiten buscar por diseños cualitativos con- cretos, $\mathrm{u}$ otras como CUIDEN permiten hacer búsquedas específicas en investigación cualitativa con la opción CUIDENcualitativa.

¿Qué diseños cualitativos incluir?

Esta pregunta es difícil de responder si nos atenemos a la diversidad de propuestas que podemos encontrar entre los autores que han trabajado la Metasíntesis. De un modo operativo, podemos concluir que la pregunta de investigación es la que marcará la elección de los diseños. Por ejemplo, si nuestra pregunta tiene que ver con la experiencia y vivencia de las personas ante una situación de salud o enfermedad, lo recomendado sería seleccionar diseños fenomenológicos; si pretendemos describir aspectos culturales, probablemente los diseños etnográficos son los que más nos pueden ayudar. También podríamos metateorizar a partir de estudios de teoría fundamentada. En síntesis, las posibilidades son varias $y$, en este sentido, tampoco podemos adoptar una actitud excluyente ya que cualquiera de los diseños que ofrece la investigación cualitativa original aporta hallazgos que son susceptibles de ser metasintetizados, por lo que dependerá de la amplitud y del interés de la pregunta. Ahora bien, también se ha de tener en cuenta que combinar estudios con diferentes enfoques metodológicos puede hacer más complejo el procedimiento de síntesis.

¿Cómo leer de manera crítica la investigación cualitativa?

Conviene tener claro que la lectura crítica implica un análisis de la calidad metodológica y de la relevancia de los hallazgos. Es decir, no se trata simplemente de ver que un estudio cumple con lo que se espera que debe cumplir desde el punto de vista de la metodología cualitativa, sino que además procede hacer una 
análisis profundo de los hallazgos que presenta, con objeto de verificar si efectivamente contribuyen de forma significativa al integrarlos junto a otros. Sandelosky y Barroso (2003), autoras referentes en Metasíntesis, ponen el énfasis precisamente en la relevancia de los hallazgos y no tanto en el criterio de calidad.

Existen diversas guías de ayuda para la lectura crítica de estudios cualitativos. Es de especial interés la guía "10 preguntas para ayudarte a entender un estudio cualitativo" de CASPE (Cano, González \& Cabello López, 2010) que incide tanto en la calidad como en la relevancia de los hallazgos del estudio. También destaca la guía COREQ (Tong, Sainsbury \& Craig, 2007) centrada fundamentalmente en el análisis metodológico. Al igual que en otros procedimientos análogos de síntesis del conocimiento, se recomienda emplear algún sistema numérico que permita puntuar el estudio y establecer un mínimo necesario consensuado por el equipo. Del mismo modo, conviene que la revisión de cada estudio se haga por pares y en caso de discrepancia, un tercer revisor intervenga para resolverla.

¿Cómo analizar los datos?.

Las técnicas posibles para sintetizar los datos son variadas. Podemos optar por un procedimiento sencillo de síntesis temática de tipo narrativo, que vaya mostrando de forma integrada los principales hallazgos encontrados en los estudios analizados, o bien trabajar más profundamente los datos incorporando procedimientos propios de análisis cualitativo. El análisis lo podemos enfocar desde planteamientos teóricos previos, de modo que busquemos en los hallazgos de los estudios seleccionados aquellos aspectos enmarcados en esos planteamientos, o bien optar por un procedimiento ortodoxo donde las categorías que estructuran los resultados emerjan en el análisis. También es posible el empleo de enfoques mixtos.

Una cuestión a considerar se relaciona con la forma en la que se presentan los resultados en los estudios cualitativos, especialmente en el campo de las Ciencias de la Salud. Así, podemos encontrar los resultados en un capítulo independiente o bien junto con la discusión. En cualquiera de los casos, es conveniente diferenciar en unidades de significado los resultados en sí del estudio de las interpretaciones de los investigadores, que pueden ser tratadas como notas complementarias (tipo memos) que también ayudarán en la Metasíntesis. El uso de software de análisis cualitativo facilita bastante estos procedimientos.

Finalmente, conviene señalar que hay procedimientos analíticos de tipo cuantitativo que son útiles. Por ejemplo, el empleo del análisis de contenido cuantitativo nos puede permitir evaluar la frecuencia de aparición de un concepto o de una idea; también interesante es el cálculo del "effect size" propuesto por Sandelowsky, Barroso y Corrine (2007): se trata de un indicador útil para describir la frecuencia con la que un hallazgo aparece en el total de estudios incluidos en la Metasíntesis.

\section{Consideraciones finales}

El objetivo de este editorial ha sido plantear algunas reflexiones de tipo conceptual y metodológico en torno a los procedimientos de Metasíntesis. Conviene destacar la oportunidad que supone este diseño tanto por lo que enriquece la metodología de investigación en Ciencias de la Salud, como por las implicaciones prácticas que se derivan de sus resultados, que ponen énfasis en la perspectiva humanista del cuidado al tomar como fuente de datos la investigación cualitativa. 
Desde el punto de vista metodológico, son diseños complejos que requieren la exhaustividad característica de la investigación secundaria y que incorpora matices que son propios del paradigma en el que se ubican. Conviene tener presente que sus procedimientos suponen un valor en sí mismo, por lo que es un diseño trasladable a otros paradigmas o perspectivas en investigación.

\section{Bibliografía}

- Balmer, C., Griffiths, F. \& Dunn, J. (2014). A qualitative systematic review exploring lay understanding of cancer by adults without a cancerdiagnosis. J Adv Nurs, 70(8), 1688-701.

- Blanca-Gutiérrez, J.J., Linares-Abad, M., Grande-Gascón, M.L., Jiménez-Díaz, M.C. \& Hidalgo-Pedraza, L. (2012). Relación del cuidado que demandan las personas mayores en hogares para ancianos: metaestudio cualitativo. Aquichan, 12 (3), 213-227

- Boyce, M.B., Browne, J.P. \& Greenhalgh, J. (2014). The experiences of professionals with using information from patient-reported outcome measures to improve the quality of healthcare: a systematic review of qualitative research. BMJ Qual Saf, 23(6), 508-18.

- Cano Arana, A., González Gil, T. \& Cabello López, J.B. (2010). Plantilla para ayudarte a entender un estudio cualitativo. En: CASPe. Guías CASPe de Lectura Crítica de la Literatura Médica. Alicante, CASPe, p.3-8.

- Capilla Díaz, C., Bonill de las Nieves, C., Montoya Suárez, R., Gálvez González, M., Martín Muñoz, B. ... Hueso Montoro, C. (2014). La experiencia de portar un estoma digestivo y su relación con metodología enfermera. Metaestudio cualitativo e implementación de evidencias $Q$ a través de vías clínicas. Biblioteca Lascasas, 10(1), recuperado de: http://www.index-f.com/lascasas/ documentos/1c0745.php

- Capilla-Díaz, C., Black, P., Bonill-de las Nieves, C., Gómez-Urquiza, J.L., Hernández Zambrano, S.M., Montoya-Juárez, R., ... Hueso-Montoro, C. (2016). The patient experience of having a stoma and its relation to nursing practice: implementation of qualitative evidence through clinical pathways. Gastrointestinal Nursing, 14 (3), 39-46.

- Carrillo-González, G.M., Gómez-Ramírez, O.J. \& Vargas-Rosero, E. (2007). La Metasíntesis: una Metodología de Investigación. Rev. salud pública, 9(4), 609-617.

- Chen, C.M., \& Chang Yeh, M. (2015). The experiences of diabetics on self-monitoring of blood glucose: a qualitative metasynthesis. J Clin Nurs, 24(5-6), 614-26.

- Hernández Zambrano S.M., Hueso Montoro, C., Montoya Juárez, R., Gómez Urquiza, J.L. \& Bonill de las Nieves, C. (2016). Metaestudio cualitativo sobre vivencias y gestión del cotidiano en adultos mayores que padecen enfermedades crónicas. Cultura de los cuidados, (44), 75-90.

- Higgins, J.P.T. \& Green, S. (2011). Cochrane Handbook for Systematic Reviews of Interventions Version 5.1.0 The Cochrane Collaboration, recuperado de: http://handbook.cochrane.org/

- Luengo González, R. (2010). Herramientas de ayuda a la toma de decisiones clínicas para Enfermería: las Guías de Práctica Clínica y otros recursos basados en la Evidencia. Evidentia, 7(32), recuperado de: http://www. index-f.com/evidentia/n32/ev3208.php

- Mahtani Chugani, V., Axpe Caballero, M.A., Serrano Aguilar, P., González Castro, I. \& Fernández Vega, E. (2007). Metodología para incorporar los estudios cualitativos en la evaluación de tecnologías sanitarias. Santa Cruiz de Tenerrige, Servicio Canario de Salud.

- Sandelowski, M. \& Barroso, J. (2003). Toward a metasynthesis of qualitative findings on motherhood in HIV-positive women. Res Nurs Health, 26(2), 153-70.

- Sandelowski, M., Barroso, J. \& Corrine, I. (2007). Using Qualitative Metasummary to Synthesize Qualitative and Quantitative Descriptive Findings. Res Nurs Health, 30(1), 99-111.

- Tong, A., Sainsbury, P. \& Craig, J. (2007) Consolidated criteria for reporting qualitative research (COREQ): a 32-item checklist for interviews and focus groups. Int $J$ Qual Health Care, 19(6), 349-357. 